\title{
Validity of Swanepoel's Method for Calculating the Optical Constants of Thick Films
}

\author{
E.R. ShaABAN ${ }^{a}$, I.S. YAhia ${ }^{b, c, *}$ AND E.G. El-Metwally ${ }^{c}$ \\ ${ }^{a}$ Department of Physics, Faculty of Science, Al-Azahar University, Assiut, 71542, Egypt \\ ${ }^{b}$ Nano-Science lab, Physics Department, Faculty of Education, Ain Shams University, Roxy, Cairo, Egypt \\ ${ }^{c}$ Semiconductor Laboratory, Physics Department, Faculty of Education, Ain Shams University, Roxy, Cairo, Egypt
}

(Received February 25, 2011; in final form May 21, 2011)

\begin{abstract}
Optical constants, dispersion and oscillator parameters of different thicknesses of amorphous $\mathrm{Ge}_{25} \mathrm{Cd}_{5} \mathrm{Se}_{70}$ films have been deposited onto glass substrates using thermal evaporation technique. The optical constants have been investigated by optical spectrophotometry measurements. The straight forward analysis proposed by Swanepoel, which is based on the use of the extremes of the interference fringes has been used in order to derive the refractive index and the film thickness in $\mu \mathrm{m}$ range. The refractive index could be extrapolated by the Cauchy dispersion relationship over the whole spectral range, which extended from 400 to $2500 \mathrm{~nm}$. It is observed that, refractive index $\mathrm{n}$ increases with the film thickness. The possible optical transition is found to be allowed indirect transition with energy gap increase from 1.915 to $1.975 \mathrm{eV}$ with increasing film thickness. The dispersion of the refractive index is discussed in terms of the Wemple-DiDomenico single oscillator model. The interband oscillator wavelength, the average oscillator strength, and the optical conductivity were estimated for different thicknesses of amorphous $\mathrm{Ge}_{25} \mathrm{Cd}_{5} \mathrm{Se}_{70}$ films.
\end{abstract}

PACS: 68.35.bj , 61.05.cp, 78.20.Ci

\section{Introduction}

Chalcogenides have recently attracted the attention of solid-state physicists, chemists and electronic engineers on account of their potential application in various solid-state devices. Chalcogenide glasses are important materials due to their potential use in integrated optics, optical imaging, optical data storage, and infrared optics [1-3]. They are attracting an extensive attention due to their practical and potential uses in the civil, medical and military areas, especially in the fields of infrared optics, opto-electronics, photonics, fiber optics, and novel memory devices [4]. High attention has been paid to the influence of impurities on the structural and optical properties of chalcogenide glasses and this influence can be quite different for different impurities. Optical properties of chalcogenides thin films have been the subject of intense study during the last decades, and great efforts have been made to develop the mathematical formulation describing the transmittance and reflectance of different optical systems [5-13]. Chalcogenide glasses are well-known IR-transmitting materials [14]. They exhibit a wide range of photoinduced effects accompanied by significant changes in their optical constants, that enable them to be used as optical recording or imaging media [15], absorption filters, and many other optical elements [16]. Knowledge

\footnotetext{
* corresponding author; e-mail: dr_isyahia@yahoo.com
}

of the optical properties of amorphous materials are obviously necessary for exploiting these materials in very interesting potential technologies.

The optical features of semiconductor films depend on different parameters, such as conditions of their preparations, film thickness and deposition rate. This paper aims to investigate the effect of film thickness on the optical properties of amorphous $\mathrm{Ge}_{25} \mathrm{Cd}_{5} \mathrm{Se}_{70}$ alloy system.

The straightforward method proposed by Swanepoel [6], which is based on the use of the extremes of the interference fringes of transmission spectrum alone, will be used in order to derive the real and imaginary parts of the complex index and also the film thickness for the amorphous $\mathrm{Ge}_{25} \mathrm{Cd}_{5} \mathrm{Se}_{70}$ semiconductor films.

The main purpose of this manuscript is how can we apply Swanepoel's method rigorously in sample with higher thin film thickness in the range about 0.651 to $2.346 \mu \mathrm{m}$. According to our knowledge, no authors applied this method for the higher thin film thickness. The objective of the present work is twofold: the first is to determine the optical constants of uniform amorphous $\mathrm{Ge}_{25} \mathrm{Cd}_{5} \mathrm{Se}_{70}$ films using their optical transmission and reflection spectrum with high precision when we change the film thickness of $\mathrm{Ge}_{25} \mathrm{Cd}_{5} \mathrm{Se}_{70}$. The second is to estimate both dispersion and oscillator parameters of different thicknesses of amorphous $\mathrm{Ge}_{25} \mathrm{Cd}_{5} \mathrm{Se}_{70}$ films. 


\section{Experimental work}

Bulk chalcogenide $\mathrm{Ge}_{25} \mathrm{Cd}_{5} \mathrm{Se}_{70}$ sample was prepared according to the conventional melt-quenched technique. The high-purity elements were weighted and placed together in a pre-cleaned and outgassed silica ampoule, which has been evacuated to a pressure of about $2 \times$ $10^{-5}$ Torr and then, sealed. The synthesis was performed in a rocking furnace at a temperature of approximately $1223 \mathrm{~K}$, for about $24 \mathrm{~h}$. hand shaking of the constituent materials inside the ampoule in the furnace was necessary to realize the homogeneity of the composition; and then the ampoule was quenched in a ice water bath to avoid crystallization. To avoid the substrate contamination, the glass substrates were cleaned using detergent solution, followed by multiple rinsing in boiling distilled water to remove traces of detergent. The substrates were then cleaned in an ultrasonic cleaner for 15 min and subsequently dried in flowing hot air. The thin films were deposited by evaporating the bulk chalcogenide sample onto pre-cleaned glass substrates kept at room temperature, using a conventional coating unit (Edwards coating system E-306) under vacuum of about $2 \times 10^{-5}$ Torr. The evaporation rate as well as the film thickness was controlled using a thickness monitor Edward FTM5. The composition of the investigated thin films deposited were checked using the energy dispersive X-ray spectroscopy (EDX) using scanning electron microscope (JEOL-JSM 5400) with EDX unit (Oxford). Fully quantitative analysis results were obtained from the spectra by processing the data through ZAF correction program. It was found that the compositions of the investigated thin films are approximately stoichiometrically closer to the starting bulk composition within $1 \%$.

The mechanical rotation of the substrate holder $(\approx 30 \mathrm{rpm})$ was done during the deposition for producing a homogeneous thin film. The deposition rate was at $15 \AA / \mathrm{s}$. Such a low deposition rate produces a film composition, which is very close to that of the bulk starting material [17]. Four different film thicknesses were deposited which are referrred as A1, A2, A3, and A4.

The structure of the as-deposited films were checked at room temperature by means of X-ray powder diffraction (XRD) Philips diffractometry (1710), with $\mathrm{Cu} K_{\alpha_{1}}$ radiation $(\lambda=1.54056 \AA)$. The data collection was performed by step scan mode, in a $2 \theta$ range between $10^{\circ}$ and $70^{\circ}$ with step-size of $0.02^{\circ}$ and step time of $0.6 \mathrm{~s}$. Pure silicon $\mathrm{Si} \approx 99.9999 \%$ was used as an internal standard.

There are many different techniques for determining refractive index, $n$, and absorption coefficient, $\alpha$, of a thin semiconducting film from spectrophotometer data. One of the earliest methods involves measuring reflections and transmission at the same location. Special modifications to the spectrophotometer are required and, unless the sample is optically flat and parallel, it is vital that in each measurement we illuminate exactly the same spot in order to avoid errors being introduced from sample inhomogeneities [13]. This paper is concerned with the optical properties of uniform amorphous $\mathrm{Ge}_{25} \mathrm{Cd}_{5} \mathrm{Se}_{70}$ films using their optical transmission and reflection spectrum. Optical characterization of the presence films has been carried out from the spectral transmittance and reflectance, which were obtained through JASCO V-570 double beam spectrophotometer. The measurements have been performed in the wavelength region from 400 to $2500 \mathrm{~nm}$. The transmittance and reflectance measurements were performed at normal incidence.

\section{Theoretical considerations}

Consider the optical system consisting of $\mathrm{As}_{2} \mathrm{~S}_{3}$ thin films evaporated onto thick, finite, transparent substrates. The homogeneous film has thickness $d$ and complex refractive index $n=n-\mathrm{i} k$, where $n$ is the refractive index and $k$ - the extinction coefficient, which can be expressed in terms of the absorption coefficient $\alpha$ by the equation: $k=\alpha \lambda / 4 \pi$. The thickness of the substrate is several orders of magnitude larger than $d$ and its index of refraction is $s$. The system is surrounded by air with refractive index $n_{0}=1$. Taking all the multiple reflections at the three interfaces into account, it can be shown that in the case $k^{2} \ll n^{2}$ the expression for the transmittance $T$ for normal incidence is given by [9-11]:

$$
\left.T(\lambda, s, n, d, k)\right|_{k=0}=\frac{A \chi_{\alpha}}{B-C \chi_{a} \cos \varphi+D \chi_{a}^{2}},
$$

where $A=16 n^{2} s, B=(n+1)^{3}\left(n+s^{2}\right), C=2\left(n^{2}-\right.$ 1) $\left(n^{2}-s^{2}\right), D=(n-1)^{3}\left(n-s^{2}\right), \varphi=4 \pi n d / \lambda$ and $\chi_{a}(\lambda)$, the absorbance, is given by the formula $\chi_{a}=\exp (-\alpha d)$. Moreover, the values of the transmission at the extremes of the interference fringes can be obtained from Eq. (1) by setting the interference condition $\cos \phi=+1$ for maxima and $\cos \phi=-1$ for minima. From these two new formulae, many of the equations that provide the basis of the method in use are easily derived [7].

\section{Results and discussion}

XRD analysis results of the as-deposited films of $\mathrm{Ge}_{25} \mathrm{Cd}_{5} \mathrm{Se}_{70}$ grown on polished fused silica glass substrate are represented in Fig. 1. The XRD patterns demonstrate that the films exhibit mainly amorphous structure and do not exhibit any peaks for crystalline phase.

The variation of absolute value of normal incidence transmittance $T(\lambda)$ and reflectance spectra $R(\lambda)$ versus wavelength $\lambda$ of $\mathrm{Ge}_{25} \mathrm{Cd}_{5} \mathrm{Se}_{70}$ glasses thin films on fused silica glass substrate is shown in Fig. 2. The spectra of all films are found to be similar with relative transmission $85-60 \%$ and slightly higher transmission envelope. Figure 3 shows the two typical spectral transmittances of $\mathrm{A} 1$ and $\mathrm{A} 4$ amorphous $\mathrm{Ge}_{25} \mathrm{Cd}_{5} \mathrm{Se}_{70}$ thin films.

Both refractive index $n$ and film thickness of different thickness $\mathrm{Ge}_{25} \mathrm{Cd}_{5} \mathrm{Se}_{70}$ thin films can be calculated as follows:

1. The necessary values of the refractive index of the substrate, $s$ are obtained from the transmission spectrum 


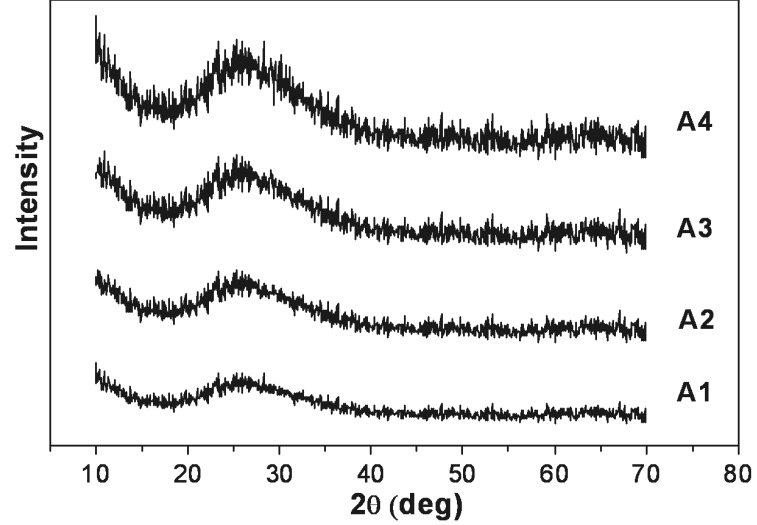

Fig. 1. X-ray diffraction patterns for the as prepared $\mathrm{Ge}_{25} \mathrm{Cd}_{5} \mathrm{Se}_{70}$ thin films with different thickness.

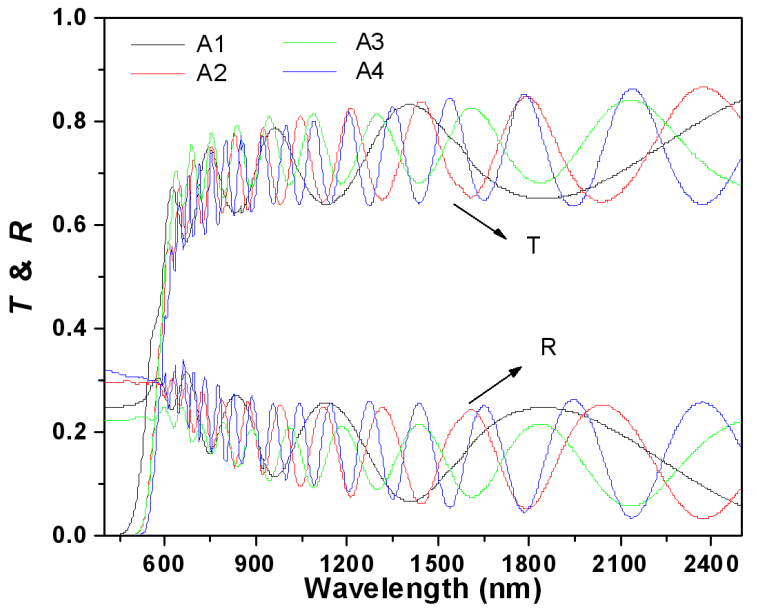

Fig. 2. Transmittance $T(\lambda)$ and reflectance $R(\lambda)$ spectra of four different thickness A1, A2, A3, and A4 of $\mathrm{Ge}_{25} \mathrm{Cd}_{5} \mathrm{Se}_{70}$ thin films deposited onto transparent substrates.

of the substrate $T_{\mathrm{s}}$ using the well-known equation

$$
s=\frac{1}{T_{\mathrm{s}}}+\left(\frac{1}{T_{\mathrm{s}}}-1\right)^{\frac{1}{2}} .
$$

2. In terms of Swanepoel's method, which is based on the idea of Manifacier et al. [18] of creating the upper and lower envelopes of the transmittance spectrum (see Fig. 2), the refractive index of the film $n_{1}$ in the spectral region of transparent, weak and medium absorption regions can be calculated by the expression

$$
n_{1}=\left[N_{1}+\left(N_{1}^{2}-s^{2}\right)^{\frac{1}{2}}\right]^{\frac{1}{2}},
$$

where

$$
N_{1}=\frac{2 s}{T_{\mathrm{m}}}+\frac{s^{2}+1}{2}
$$

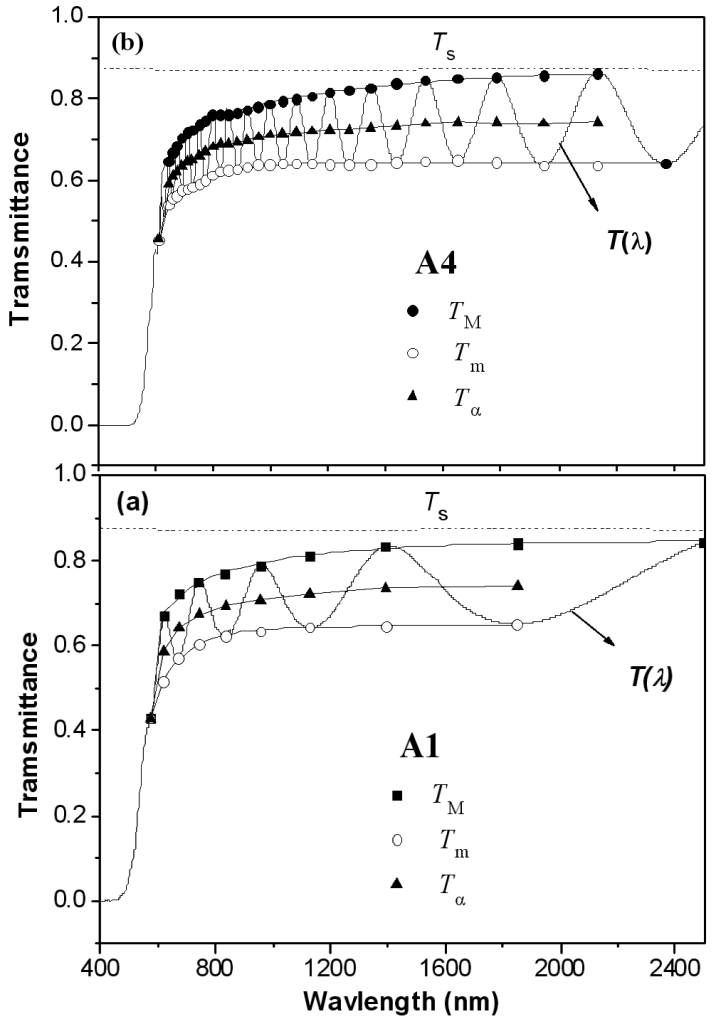

Fig. 3. Two typical transmission spectra for two samples $\mathrm{A} 1$ and $\mathrm{A} 4$ of amorphous $\mathrm{Ge}_{25} \mathrm{Cd}_{5} \mathrm{Se}_{70}$ thin films. Curves $T_{\mathrm{s}}, T_{\mathrm{M}}, T_{\alpha}$, and $T_{\mathrm{m}}$, according to the text.

$$
N_{1}=2 s \frac{T_{\mathrm{M}}-T_{\mathrm{m}}}{T_{\mathrm{M}} T_{\mathrm{m}}}+\frac{s^{2}+1}{2}
$$

for weak and medium absorption regions. Here $T_{\mathrm{M}}$ and $T_{\mathrm{m}}$ are the transmission maximum and the corresponding minimum at a certain wavelength $\lambda$. Alternatively, one of these values is an experimental interference extreme and the other one is derived from the corresponding envelope; both envelopes were computer-generated using the program Origin version 7 (OriginLab Corp.). The values of the refractive index $n_{1}$, as calculated from Eq. (3) are shown in Table I. Figure 4 illustrates the first approximation values of refractive index $\left(n_{1}\right)$ dispersion spectra for four different thickness $\mathrm{A} 1, \mathrm{~A} 2, \mathrm{~A} 3$, and $\mathrm{A} 4$ of $\mathrm{Ge}_{25} \mathrm{Cd}_{5} \mathrm{Se}_{70}$ thin films.

3 . If $n_{\mathrm{e} 1}$ and $n_{\mathrm{e} 2}$ are the refractive indices at two adjacent maxima (or minima) at $\lambda_{1}$ and $\lambda_{2}$, it follows that the film thickness is given by the expression:

$$
d=\frac{\lambda_{1} \lambda_{2}}{2\left(\lambda_{1} n_{\mathrm{e} 2}-\lambda_{2} n_{\mathrm{e} 1}\right)} .
$$

The values of thickness $d$ of the studied films determined by this equation are listed as $d_{1}$ in Table I. The average values of $d_{1}$, corresponding to each thickness at different values of maxima and minima of the transmittance curve are listed in Table I.

for transparent region and 
TABLE I

Values of $\lambda, T_{\mathrm{M}}$ and $T_{\mathrm{m}}$ for the four different thickness of amorphous $\mathrm{Ge}_{25} \mathrm{Cd}_{5} \mathrm{Se}_{70}$ thin films corresponding to transmission spectra. The calculated values of refractive index and film thickness are based on the envelope method.

\begin{tabular}{|c|c|c|c|c|c|c|c|c|c|}
\hline$\lambda$ & $T_{\mathrm{M}}$ & $T_{\mathrm{m}}$ & $s$ & $n_{1}$ & $d_{1}[\mathrm{~nm}]$ & $m_{0}$ & $m$ & $d_{2}[\mathrm{~nm}]$ & $n_{2}$ \\
\hline \multicolumn{10}{|c|}{ Sample A1 } \\
\hline 578 & 0.667 & 0.494 & 1.527 & 2.485 & - & 5.797 & 5.5 & 639.6 & 2.441 \\
\hline 620 & 0.701 & 0.541 & 1.53 & 2.342 & 522.7 & 5.093 & 5 & 661.9 & 2.38 \\
\hline 674 & 0.73 & 0.579 & 1.533 & 2.253 & 613.6 & 4.507 & 4.5 & 673.1 & 2.329 \\
\hline 744 & 0.754 & 0.607 & 1.536 & 2.204 & 685.4 & 3.994 & 4 & 675.1 & 2.285 \\
\hline 836 & 0.776 & 0.626 & 1.539 & 2.185 & 737 & 3.523 & 3.5 & 669.6 & 2.246 \\
\hline 958 & 0.795 & 0.638 & 1.54 & 2.188 & 756.3 & 3.079 & 3 & 656.8 & 2.207 \\
\hline 1130 & 0.812 & 0.644 & 1.539 & 2.206 & 729 & 2.632 & 2.5 & 640.3 & 2.169 \\
\hline 1394 & 0.828 & 0.646 & 1.532 & 2.228 & 674.9 & 2.155 & 2 & 625.8 & 2.14 \\
\hline 1850 & 0.84 & 0.647 & 1.52 & 2.241 & - & 1.633 & 1.5 & 619.1 & 2.131 \\
\hline \multicolumn{10}{|c|}{$\bar{d}_{1}=674 \mathrm{~nm}, \sigma_{1}=82 \mathrm{~nm}(12 \%), \bar{d}_{2}=651 \mathrm{~nm}, \sigma_{2}=20 \mathrm{~nm}(3.1 \%)$} \\
\hline \multicolumn{10}{|c|}{ Sample A2 } \\
\hline 634 & 0.814 & 0.587 & 1.536 & 2.423 & - & 11.262 & 11 & 1439 & 2.392 \\
\hline 662 & 0.819 & 0.602 & 1.538 & 2.377 & 1207 & 10.581 & 10.5 & 1462 & 2.384 \\
\hline 688 & 0.822 & 0.613 & 1.539 & 2.344 & 1366 & 10.041 & 10 & 1468 & 2.36 \\
\hline 718 & 0.826 & 0.623 & 1.54 & 2.315 & 1353 & 9.503 & 9.5 & 1473 & 2.339 \\
\hline 754 & 0.829 & 0.632 & 1.542 & 2.29 & 1407 & 8.952 & 9 & 1481 & 2.327 \\
\hline 792 & 0.833 & 0.639 & 1.543 & 2.272 & 1462 & 8.456 & 8.5 & 1481 & 2.309 \\
\hline 838 & 0.836 & 0.645 & 1.544 & 2.259 & 1522 & 7.944 & 8 & 1484 & 2.299 \\
\hline 886 & 0.839 & 0.65 & 1.545 & 2.251 & 1585 & 7.487 & 7.5 & 1476 & 2.279 \\
\hline 944 & 0.843 & 0.653 & 1.545 & 2.247 & 1555 & 7.014 & 7 & 1471 & 2.266 \\
\hline 1012 & 0.846 & 0.655 & 1.545 & 2.246 & 1608 & 6.54 & 6.5 & 1465 & 2.256 \\
\hline 1086 & 0.85 & 0.657 & 1.545 & 2.247 & 1551 & 6.098 & 6 & 1450 & 2.235 \\
\hline 1186 & 0.853 & 0.658 & 1.543 & 2.25 & 1522 & 5.59 & 5.5 & 1450 & 2.237 \\
\hline 1294 & 0.857 & 0.658 & 1.54 & 2.252 & 1479 & 5.129 & 5 & 1436 & 2.219 \\
\hline 1446 & 0.86 & 0.658 & 1.536 & 2.254 & 1484 & 4.594 & 4.5 & 1443 & 2.232 \\
\hline 1606 & 0.862 & 0.659 & 1.531 & 2.254 & 1528 & 4.136 & 4 & 1425 & 2.203 \\
\hline 1830 & 0.865 & 0.659 & 1.526 & 2.254 & - & 3.63 & 3.5 & 1421 & 2.197 \\
\hline \multicolumn{10}{|c|}{$\bar{d}_{1}=1474 \mathrm{~nm}, \sigma_{1}=108 \mathrm{~nm}(7.3 \%), \bar{d}_{2}=1458 \mathrm{~nm}, \sigma_{2}=21 \mathrm{~nm}(1.4 \%)$} \\
\hline \multicolumn{10}{|c|}{ Sample A3 } \\
\hline 626 & 0.761 & 0.545 & 1.536 & 2.486 & - & 12.741 & 12.5 & 1574 & 2.442 \\
\hline 648 & 0.769 & 0.559 & 1.537 & 2.443 & 1326 & 12.095 & 12 & 1592 & 2.427 \\
\hline 670 & 0.777 & 0.572 & 1.538 & 2.408 & 1451 & 11.531 & 11.5 & 1600 & 2.405 \\
\hline 694 & 0.783 & 0.583 & 1.539 & 2.377 & 1420 & 10.99 & 11 & 1606 & 2.383 \\
\hline 724 & 0.79 & 0.595 & 1.541 & 2.347 & 1459 & 10.401 & 10.5 & 1620 & 2.373 \\
\hline 754 & 0.796 & 0.604 & 1.542 & 2.324 & 1538 & 9.89 & 10 & 1622 & 2.353 \\
\hline 790 & 0.802 & 0.613 & 1.543 & 2.304 & 1574 & 9.358 & 9.5 & 1629 & 2.342 \\
\hline 828 & 0.808 & 0.62 & 1.544 & 2.289 & 1609 & 8.871 & 9 & 1628 & 2.326 \\
\hline 874 & 0.814 & 0.627 & 1.545 & 2.277 & 1625 & 8.361 & 8.5 & 1631 & 2.319 \\
\hline 924 & 0.819 & 0.632 & 1.545 & 2.27 & 1706 & 7.883 & 8 & 1628 & 2.307 \\
\hline 980 & 0.824 & 0.636 & 1.545 & 2.266 & 1719 & 7.421 & 7.5 & 1622 & 2.294 \\
\hline 1046 & 0.83 & 0.64 & 1.545 & 2.266 & 1693 & 6.95 & 7 & 1616 & 2.285 \\
\hline 1124 & 0.835 & 0.642 & 1.544 & 2.267 & 1711 & 6.473 & 6.5 & 1611 & 2.28 \\
\hline 1212 & 0.841 & 0.644 & 1.542 & 2.271 & 1735 & 6.012 & 6 & 1601 & 2.27 \\
\hline 1316 & 0.846 & 0.645 & 1.54 & 2.275 & 1735 & 5.548 & 5.5 & 1591 & 2.259 \\
\hline 1438 & 0.851 & 0.646 & 1.536 & 2.28 & 1641 & 5.087 & 5 & 1577 & 2.244 \\
\hline 1604 & 0.857 & 0.647 & 1.531 & 2.285 & 1637 & 4.57 & 4.5 & 1580 & 2.253 \\
\hline 1788 & 0.861 & 0.647 & 1.527 & 2.289 & 1696 & 4.107 & 4 & 1562 & 2.232 \\
\hline 2032 & 0.865 & 0.647 & 1.525 & 2.295 & - & 3.624 & 3.5 & 1549 & 2.22 \\
\hline
\end{tabular}


TABLE I (cont.)

\begin{tabular}{c|c|c|c|c|c|c|c|c|c}
\hline \hline$\lambda$ & $T_{\mathrm{M}}$ & $T_{\mathrm{m}}$ & $s$ & $n_{1}$ & $d_{1}[\mathrm{~nm}]$ & $m_{0}$ & $m$ & $d_{2}[\mathrm{~nm}]$ & $n_{2}$ \\
\hline \multicolumn{7}{c}{ Sample A 4} \\
\hline 648 & 0.758 & 0.526 & 1.526 & 2.555 & - & 18.396 & 18 & 2282 & 2.486 \\
662 & 0.764 & 0.537 & 1.527 & 2.517 & 1861 & 17.738 & 17.5 & 2301 & 2.469 \\
676 & 0.769 & 0.547 & 1.528 & 2.484 & 1822 & 17.143 & 17 & 2313 & 2.449 \\
694 & 0.774 & 0.559 & 1.529 & 2.448 & 1801 & 16.458 & 16.5 & 2339 & 2.44 \\
712 & 0.78 & 0.569 & 1.53 & 2.419 & 1921 & 15.848 & 16 & 2355 & 2.428 \\
732 & 0.785 & 0.578 & 1.53 & 2.392 & 1952 & 15.243 & 15.5 & 2372 & 2.418 \\
754 & 0.79 & 0.587 & 1.531 & 2.368 & 2087 & 14.653 & 15 & 2388 & 2.41 \\
776 & 0.795 & 0.595 & 1.532 & 2.35 & 2240 & 14.126 & 14.5 & 2394 & 2.398 \\
800 & 0.8 & 0.601 & 1.533 & 2.334 & 2301 & 13.611 & 14 & 2399 & 2.387 \\
826 & 0.805 & 0.608 & 1.533 & 2.322 & 2378 & 13.112 & 13.5 & 2402 & 2.376 \\
854 & 0.81 & 0.613 & 1.534 & 2.312 & 2393 & 12.63 & 13 & 2401 & 2.366 \\
886 & 0.815 & 0.618 & 1.534 & 2.305 & 2424 & 12.137 & 12.5 & 2402 & 2.36 \\
920 & 0.819 & 0.622 & 1.535 & 2.301 & 2481 & 11.668 & 12 & 2399 & 2.353 \\
958 & 0.824 & 0.625 & 1.535 & 2.299 & 2542 & 11.197 & 11.5 & 2396 & 2.348 \\
998 & 0.829 & 0.627 & 1.535 & 2.3 & 2619 & 10.75 & 11 & 2387 & 2.339 \\
1042 & 0.834 & 0.629 & 1.535 & 2.302 & 2642 & 10.306 & 10.5 & 2377 & 2.331 \\
1090 & 0.838 & 0.631 & 1.534 & 2.305 & 2634 & 9.867 & 10 & 2364 & 2.323 \\
1144 & 0.843 & 0.632 & 1.533 & 2.31 & 2563 & 9.421 & 9.5 & 2352 & 2.316 \\
1206 & 0.848 & 0.633 & 1.532 & 2.316 & 2570 & 8.957 & 9 & 2344 & 2.313 \\
1272 & 0.852 & 0.634 & 1.53 & 2.321 & 2577 & 8.513 & 8.5 & 2329 & 2.304 \\
1348 & 0.857 & 0.634 & 1.528 & 2.327 & 2463 & 8.052 & 8 & 2317 & 2.298 \\
1438 & 0.862 & 0.634 & 1.526 & 2.332 & 2419 & 7.566 & 7.5 & 2312 & 2.298 \\
1538 & 0.866 & 0.634 & 1.523 & 2.337 & 2461 & 7.088 & 7 & 2304 & 2.294 \\
1650 & 0.87 & 0.634 & 1.519 & 2.341 & 2419 & 6.618 & 6.5 & 2291 & 2.285 \\
1786 & 0.873 & 0.634 & 1.516 & 2.344 & 2362 & 6.124 & 6 & 2285 & 2.284 \\
1946 & 0.877 & 0.634 & 1.514 & 2.349 & 2383 & 5.631 & 5.5 & 2279 & 2.281 \\
2136 & 0.88 & 0.634 & 1.516 & 2.356 & - & 5.145 & 5 & 2267 & 2.276 \\
\hline \multicolumn{7}{|c|}{$\bar{d}_{1}=2333 \mathrm{~nm}, \sigma_{1}=268 \mathrm{~nm}(11.5 \%), \bar{d}=2346 \mathrm{~nm}, \sigma_{2}=46 \mathrm{~nm}(2 \%)$} \\
\end{tabular}

TABLE II

Optical parameters of the four different thicknesses of amorphous $\mathrm{Ge}_{25} \mathrm{Cd}_{5} \mathrm{Se}_{70}$ thin films.

\begin{tabular}{c|c|c|c|c|c|c|c}
\hline \hline Samples & $E_{\mathrm{g}}^{\text {opt }}[\mathrm{eV}]$ & $E_{\mathrm{g}}^{\text {opt(WDD) }}[\mathrm{eV}]$ & $E_{0}[\mathrm{eV}]$ & $E_{\mathrm{d}}[\mathrm{eV}]$ & $n(0)$ & $\lambda_{0}[\mathrm{~nm}]$ & $S_{0}\left[\mathrm{~m}^{-2}\right]$ \\
\hline A1 & 1.915 & 2.000 & 4.000 & 14.286 & 2.138 & 310.750 & $3.698 \times 10^{13}$ \\
A2 & 1.93 & 2.048 & 4.096 & 15.752 & 2.201 & 303.494 & $4.176 \times 10^{13}$ \\
A3 & 1.961 & 2.076 & 4.152 & 16.609 & 2.236 & 299.354 & $4.464 \times 10^{13}$ \\
A4 & 1.975 & 2.093 & 4.185 & 17.44 & 2.273 & 296.979 & $4.724 \times 10^{13}$
\end{tabular}

4. By taking into account, the basic equation for the interference fringes

$$
2 n d=m \lambda \text {, }
$$

where the order number $m$ is integer for maxima and half integer for minima. This value of $m$ can now be used, along with $n_{1}$, to calculate the "order number" $m_{0}$ for the different extremes using Eq. (5).

5 . Now, the accuracy of $d$ can be significantly increased by taking the corresponding exact integer or half integer values of $m$ associated to each extreme (Fig. 2) and deriving a new thickness, $d_{2}$, from Eq. (6), again using the values of $n_{1}$, the values of $d_{2}$ found in this way have a smaller dispersion $\left(\sigma_{1}>\sigma_{2}\right)$. It should be emphasized that the accuracy of the final thickness is from about 1.5 to $3 \%$ as shown in Table I.

6. With the exact value of $m$ and the very accurate value of $d$, Eq. (7) can then be solved for $n$ at each $\lambda$ and thus, the final values of the refractive index $n_{2}$ are obtained in Table I.

It is preferred to calculate the refractive index and film thickness in uniform region of the spectra (weak and medium absorption region) and to extrapolate the refractive index in both strong absorption region and transparent region by the Cauchy equation, which is valid for the thin film model. Now, the values of $n_{2}$ can be fitted to a reasonable function such as the two-term Cauchy dispersion relationship, $n(\lambda)=a+b / \lambda^{2}$, which can be used for extrapolation of all the wavelengths [19] (see Fig. 3). The least squares fit of the two sets of values of $n_{2}$ for the different thickness samples listed in Table I, yields $n=$ $2.084+114947 / \lambda^{2}$ for sample A1, $n=2.204+89494 / \lambda^{2}$ for sample A2, $n=2.170+89283 / \lambda^{2}$ for sample A3, and $n=2.249+91611 / \lambda^{2}$ for sample A4. Figure 4 illustrates the dependence of the refractive index $n$ on the wavelength for different thicknesses of $\mathrm{Ge}_{25} \mathrm{Cd}_{5} \mathrm{Se}_{70}$ chalcogenide thin films. It is clear that the refractive index $n$ increases with the increase of the film thickness. The refractive index is related to the density and the polarizability of a given material. Thus changing the film thickness could change the density and/or the polarizability of the $\mathrm{Ge}_{25} \mathrm{Cd}_{5} \mathrm{Se}_{70}$ chalcogenide thin films. 


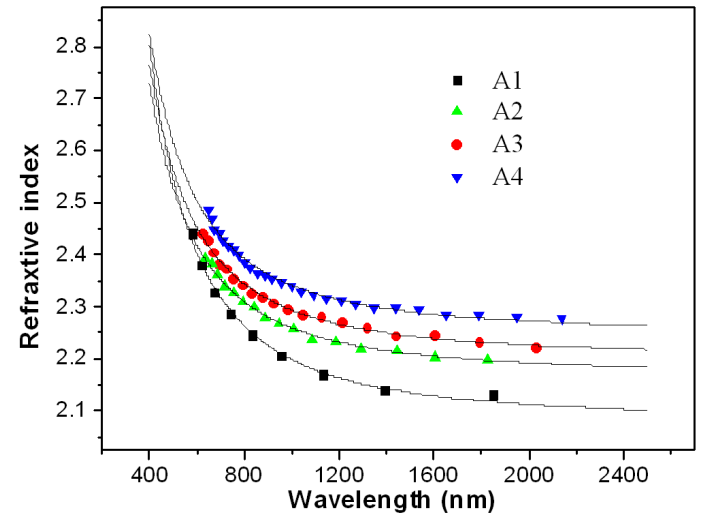

Fig. 4. Refractive index $\left(n_{2}\right)$ dispersion spectra for four different thicknesses A1, A2, A3, and A4 of $\mathrm{Ge}_{25} \mathrm{Cd}_{5} \mathrm{Se}_{70}$ thin films.

The absorption coefficient $\alpha$ can be obtained in the strong absorption region of the experimentally measured values of $R$ and $T$ according to the following expression [20]:

$$
\alpha=\frac{1}{d} \ln \left(\frac{(1-R)^{2}+\left[(1-R)^{4}+4 R^{2} T^{2}\right]^{1 / 2}}{2 T}\right),
$$

where $T$ is the transmittance, $R$ is the reflectance and $d$ is the thickness of the as-deposited films. Figure 5 shows the dependence of the absorption coefficient, $\alpha$, on the photon energy $(h \nu)$ of amorphous thin film $\mathrm{Ge}_{25} \mathrm{Cd}_{5} \mathrm{Se}_{70}$ with different thickness. It is clearly observed that the value of the absorption edge decreases as the thickness increases. It is known that in the absorption process, a photon of known energy excites an electron from lower to a higher energy state, corresponding to an absorption edge. It should be pointed out that the absorption coefficient of amorphous semiconductors, in the high-absorption region $\left(\alpha \geq 10^{4} \mathrm{~cm}^{-1}\right)$ is given according to Tauc's relation for the allowed non-direct transition [21]:

$$
\alpha(h \nu)=\frac{K\left(h \nu-E_{\mathrm{g}}^{\mathrm{opt}}\right)^{2}}{h \nu},
$$

where $K$ is constant which depends on the transition probability and $E_{\mathrm{g}}^{\mathrm{opt}}$ is the optical band gap. Figure 6 is a typical best fit of $(\alpha h \nu)^{1 / 2}$ vs. photon energy $h \nu$ for the investigated thicknesses A1, A2, A3, and A4. The values of the optical band gap $E_{\mathrm{g}}^{\mathrm{opt}}$ were taken as the intercept of $(\alpha h \nu)^{1 / 2}$ vs. $h \nu$ at $(\alpha h \nu)^{1 / 2}=0$ according to Tauc's relation in the strong-absorption region of investigated films for the allowed non-direct transition. The results indicate that the $E_{\mathrm{g}}^{\mathrm{opt}}$ value determined for different film thicknesses increase from 1.915 to $1.975 \mathrm{eV}$ with increase in the film thickness.

The energy gap values are listed in Table II. The band gap of different thickness of $\mathrm{Ge}_{25} \mathrm{Cd}_{5} \mathrm{Se}_{70}$ is lower than in $\mathrm{Se}_{70} \mathrm{Ge}_{30}(2.02 \mathrm{eV})$ [22]. Also, this confirmed that the band gap value of any composition must possess one value not dependent on the film thickness. It can be dis-

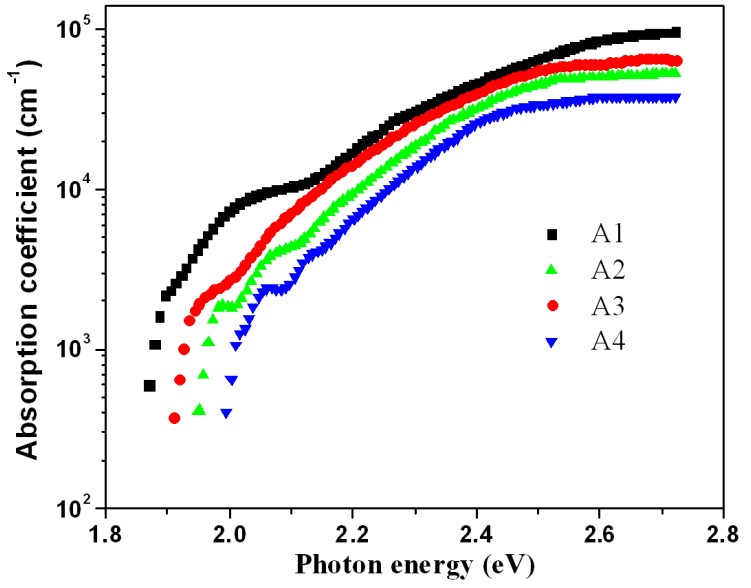

Fig. 5. The absorption coefficient against photon energy for the samples of amorphous $\mathrm{Ge}_{25} \mathrm{Cd}_{5} \mathrm{Se}_{70}$ thin films.

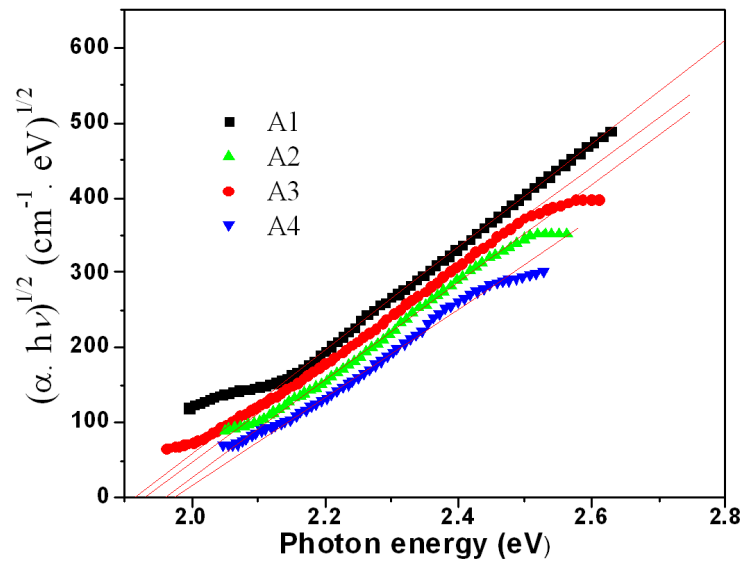

Fig. 6. The dependence of $(\alpha h \nu)^{1 / 2}$ on photon energy $h \nu$ for the different thicknesses A1, A2, A3 and A4 of amorphous $\mathrm{Ge}_{25} \mathrm{Cd}_{5} \mathrm{Se}_{70}$ thin films, from which the optical band gap $E_{\mathrm{g}}^{\mathrm{opt}}$ is estimated (Tauc's extrapolation).

cussed according to the Mott and Davis model [23, 24] that the width of localized states near the mobility edges depends on the degrees of disorder and defects presented in the amorphous structure. In particular, it is known that unsaturated bonds together with some saturated bonds are produced as a result of an insufficient number of atoms deposited in the amorphous films. The unsaturated bonds are responsible for the formation of some defects in such films [1,2]. Such defects produce localized states in the amorphous solids. So, on addition of $\mathrm{Cd}$ to Se-Ge matrix, the Cd-Se bonding is developed, which introduces a large number of defects in the formation of an impurity band adjacent to a band and formation of tails of states extending the band into the mobility gap. This led to the decrease of the band gap of different thickness $\mathrm{Ge}_{25} \mathrm{Cd}_{5} \mathrm{Se}_{70}$ than in $\mathrm{Se}_{70} \mathrm{Ge}_{30}(2.02 \mathrm{eV})$ [22]. Such decrease in the band gap can be explained also due to the 
formation of charged defect centers by the metal additives enhancing the conductivity and decreases the band gap. The bond energy between Se-Ge equals $2.12 \mathrm{eV}$ and Se-Cd equals $3.21 \mathrm{eV}$ which may be related to the decrease in the bond energy between Se (the host element) and the metal additives (Cd) [25]. It is clear from Table I that the optical bandgap increases with the increase of the film thickness. This behaviour can be explained as the presence of defects in amorphous materials [26, 27] in terms of elimination of defects in the amorphous structure. The insufficient number of atoms deposited in the amorphous film results in the existence of unsaturated bonds [28]. The unsaturated bonds are responsible for the formation of some defects in the films which produced localized states in amorphous solids [29]. Thicker films are characterized by more homogeneous network, which minimizes the number of defects and localized states, and thus the optical band gap increases [30, 31].

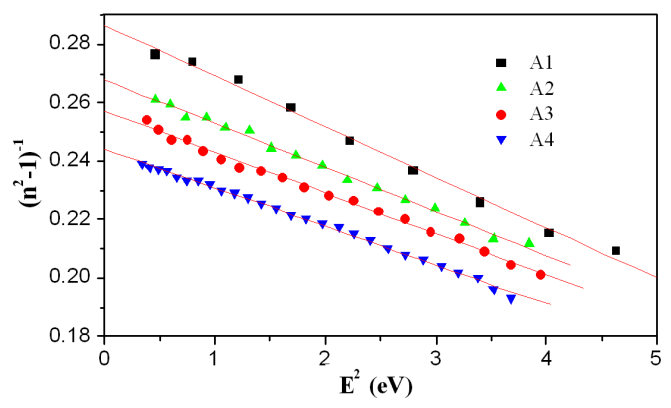

Fig. 7. Plot of refractive index factor $\left(n^{2}-1\right)^{-1}$ versus $E^{2}$ for amorphous $\mathrm{Ge}_{25} \mathrm{Cd}_{5} \mathrm{Se}_{70}$ thin films.

The dispersion plays an important role in the research for optical materials, because it is a significant factor in optical communication and in designing devices for spectral dispersion. The result of refractive index dispersion below the interband absorption edge corresponds to the fundamental electronic excitation spectrum. Thus, the data of the refractive index dispersion of the investigated compounds can be analyzed in terms of the single-effective-oscillator model proposed by the Wemple-DiDomenico (WDD) $[32,33]$. The energy dependence of $n$ of amorphous materials can be fitted to the Wemple and DiDomenico (WDD) dispersion relationship, that is single-oscillator model [24]:

$$
n^{2}(E)-1=\frac{E_{\mathrm{d}} E_{0}}{E_{0}^{2}-E^{2}},
$$

where $E_{0}$ is the single-oscillator energy and $E_{\mathrm{d}}$ - the dispersion energy. By plotting $\left(n^{2}-1\right)^{-1}$ versus $E^{2}$ (Fig. 7) and fitted the data to a straight line, $E_{0}$ and $E_{\mathrm{d}}$ can be determined from the intercept, $E_{0} / E_{\mathrm{d}}$ and the slope, $1 / E_{0} E_{\mathrm{d}}$. The values of WDD dispersion parameters, $E_{0}$ and $E_{\mathrm{d}}$ for all glass thin film samples obtained from the linear fitting are tabulated in Table II. Furthermore, as was proposed by Tanaka [34], the first approximate value of the optical band gap $E_{\mathrm{g}}^{\mathrm{opt}}$ is also derived from the
WDD model, according to the expression $E_{0} \approx 2 E_{\mathrm{g}}^{\mathrm{opt}}$, the values given in Table II. Obviously, values are almost in agreement with that obtained from the Tauc extrapolation model.

The static refractive index $n_{0}$ for the films investigated is calculated by extrapolation the WDD dispersion relation to the value of the incident photon energy $E$ approaching zero, which gives

$$
n_{0}=\left[1+\frac{E_{\mathrm{d}}}{E_{0}}\right]^{1 / 2} .
$$

The variation of the static refractive index $n_{0}$ as a function of film thickness for investigated thin films is listed in Table II.

Average interband oscillator wavelength $\left(\lambda_{0}\right)$ and the average oscillator strength $\left(S_{0}\right)$ for different thin film thicknesses were determined using the following relationship [35]:

$$
\left(\frac{n_{0}^{2}-1}{n^{2}-1}\right)=1-\left(\frac{\lambda_{0}}{\lambda}\right)^{2},
$$

where $\lambda_{0}$ values were calculated from the plots of $n^{2}-1$ vs. $\lambda^{-2}$, further, Eq. (12) can also be written as

$$
n^{2}-1=\frac{S_{0}}{1-\left(\lambda_{0} / \lambda\right)^{2}} \text {. }
$$

The $S_{0}$ values for different films were obtained using $S_{0}=\left(n_{0}^{2}-1\right) / \lambda_{0}^{2}$. The values of $\lambda_{0}$ and $S_{0}$ are summarized in Table II.

Finally, the optical conductivity is one of the powerful tools for studying the electronic states in materials [36]. If a system is subjected to an external electric field then, in general, a redistribution of charges occurs and currents are induced. For small enough fields, the induced polarization and the induced currents are proportional to the inducing field. The optical conductivity [25, 37] of the different thickness of $\mathrm{Ge}_{25} \mathrm{Cd}_{5} \mathrm{Se}_{70}$ films is calculated by

$$
\sigma=\frac{\alpha n c}{4 \pi},
$$

where $\alpha$ is the absorption coefficient, $n$ is the refractive index and $c$ is the velocity of light. Figure 8 shows the variation of the optical conductivity upon the wavelength for $\mathrm{Ge}_{25} \mathrm{Cd}_{5} \mathrm{Se}_{70}$ films. The fitting curves for both inter-

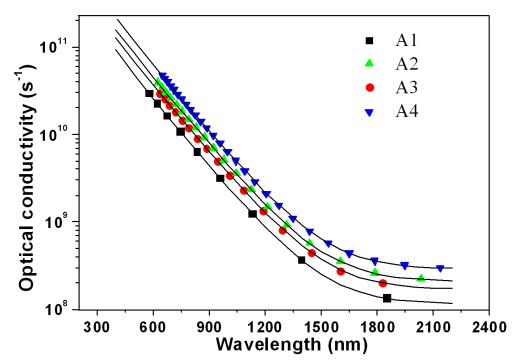

Fig. 8. The optical conductivity against photon wavelength for the samples of amorphous $\mathrm{Ge}_{25} \mathrm{Cd}_{5} \mathrm{Se}_{70}$ thin films. 
polation and extrapolation of optical conductivity can be achieved by substituting the Cauchy refractive index, which was mentioned above in Eq. (14). It is observed that the optical conductivity increases with increasing the film thickness which could be related to the increase in the refractive index and the density of localized states in the gap due to the appearance of new defects states.

\section{Conclusions}

The applied optical method makes it possible to determine the refractive index and average thickness with higher accuracy of the amorphous $\mathrm{Ge}_{25} \mathrm{Cd}_{5} \mathrm{Se}_{70}$ films of different thicknesses prepared by thermal evaporation technique. The envelope method suggested by Swanepoel has been applied rigorously to the studied films with a reasonable number of interference fringes. Swanepoel's method is valid with high precision to caulate the optical constants of the higher thin film thickness. The results indicate that the values of $n$ gradually increase with increasing film thickness, which may be related to the variation of density with thickness. The absorption coefficient and the optical band gap of the deposited films have been determined from transparency and reflectivity spectrum in the strong absorption region. The optical band gap $E_{\mathrm{g}}^{\mathrm{opt}}$ exhibits allowed indirect transitions and its value is found to increase with increasing film thickness. The dispersion parameter, the single-oscillator energy $E_{0}$, the dispersion energy $E_{\mathrm{d}}$ and the static refractive index $n_{0}$ are determined using WDD single oscillator model. The optical conductivity of the different thickness of $\mathrm{Ge}_{25} \mathrm{Cd}_{5} \mathrm{Se}_{70}$ films is calculated and its value is found to increase with the increase of the film thickness which could be related to the increase in the refractive index and the density of localized states in the gap due to the appearance of new defects states.

\section{Acknowledgments}

The authors thank Al Azhar University (Assiut, Egypt) and Ain Shams University (Cairo, Egypt) for the financial support.

\section{References}

[1] J.S. Sanghara, I.D. Agarwal, J. Non-Cryst. Solids 6, 256 (1999).

[2] K. Schwartz, The Physics of Optical Recording, Springer-Verlag, Berlin 1993.

[3] A. Bradley, Optical Storage for Computers Technology and Applications, Ellis Horwood Ltd, New York 1989.

[4] G.B. Sakr, I.S. Yahia, M. Fadel, S.S. Fouad, N. Romcevic, J. Alloys Comp. 507, 557 (2010).

[5] J.C. Manifacier, J. Gasiot, J.P. Fillard, J. Phys. E 9, 1002 (1976).

[6] R. Swanepoel, J. Phys. E, Sci. Instrum. 16, 1214 (1983).

[7] R. Swanepoel, J. Phys. E, Sci. Instrum. 17, 896 (1984).

[8] D.A. Minkov, J. Phys. D, Appl. Phys. 22, 1157 (1989).
[9] E.R. Shaaban, J. Phys. Chem. Solids 68, 400 (2007).

[10] J.J. Ruiz-Pérez, J.M. González-Leal, D.A. Minkov, E. Márquez, J. Phys. D, Appl. Phys. 34, 2489 (2001).

[11] E.R. Shaaban, M. Abdel-Rahman, El Sayed Yousef, M.T. Dessouky, Thin Solid Films 515, 3810 (2007).

[12] J.M. González-Leal, R. Prieto-Alcón, M. Stuchlik, M. Vlcek, S.R. Elliott, E. Márquez, Opt. Mater. 27, 147 (2004).

[13] E.R. Shaaban, Philos. Mag. 88, 781 (2008).

[14] J.A. Savage, Infrared Optical Materials and Their Antireflection Coatings, A. Hilger, London 1985.

[15] A.E. Owen, A.P. Firth, P.J.S. Ewen, Philos. Mag. B 52, 347 (1985).

[16] S.R. Elliott, Physics of Amorphous Materials, Longman, New York 1990.

[17] E. Marquez, A.M. Bernal-Oliva, J.M. Gonzalez-Leal, R. Prieto-Alcon, A. Ledesma, R. Jimenez-Garay, I. Martil, Mater. Chem. Phys. 60, 231 (1999).

[18] J.C. Manifacier, J. Gasiot, J.P. Fillard, J. Phys. E 9 , 1002 (1976).

[19] T.S. Moss, Optical Properties of Semiconductors, Buttenworths, London 1959.

[20] E.R. Shaaban, N. Afify, A. El-Taher, J. Alloys Comp. 482, 400 (2009).

[21] A.A. Yadav, M.A. Barote, T.V. Chavan, E.U. Masumdar, J. Alloys Comp. 509, 916 (2011).

[22] E.G. El-Metwally, M.O. Abou-Helal, I.S. Yahia, J. Ovonic Res. 4, 20 (2008).

[23] N.F. Mott, E.A. Davis, Electronic Processes in Non-Crystalline Materials, Clarendon, Oxford 1971.

[24] M. Fadel, S.A. Fayek, M.O. Abou-Helal, M.M. Ibrahim, A.M. Shakra, J. Alloys Comp. 485, 604 (2009).

[25] E.G. El-Metwally, M. Fadel, A.M. Shakra, M.A. Afifi, J. Optoelectron. Adv. Mater. 10, 1320 (2008).

[26] E.R. Shaaban, Mater. Chem. Phys. 100, 411 (2006).

[27] S. Chaudhuri, S.K. Biswas, A. Choudhury, J. Mater. Sci. 23, 4470 (1988).

[28] S.K. Biswas, S. Chaudhuri, A. Choudhury, Phys. Status Solidi A 105, 467 (1988).

[29] K.I. Arshak, C. Ahogarth, Thin Solid Films 137, 281 (1986).

[30] B.A. Mansour, H. Shaban, S.A. Gad, Y.A. El-Gendy, A.M. Salem, J. Ovonic Res. 6, 13 (2010).

[31] D.C. Sati, R. Kumar, R.M. Mehra, Turk. J. Phys. 30, 519 (2006).

[32] S.H. Wemple, M. DiDomenico, Phys. Rev. B 3, 1338 (1971).

[33] S.H. Wemple, Phys. Rev. B 7, 3767 (1973).

[34] T. Tanaka, Thin Solid Films 66, 271 (1980).

[35] N.A. Subrahamanyam, A Textbook of Optics, 9th ed., Brj Laboratory, Delhi, India 1977.

[36] T. Takami, M. Sadamichi, J. Phys. Soc. Jpn. 60, 53 (1991).

[37] P. Sharma Rangra, P. Sharma, S.C. Katyal, J. Phys. D, Appl. Phys. 41, 225307 (2008). 